\title{
Discriminative Auditory Fear Learning Requires Both Tuned and Nontuned Auditory Pathways to the Amygdala
}

\author{
Raquel Antunes and Marta A. Moita \\ Champalimaud Neuroscience Programme at Instituto Gulbenkian de Ciência, P-2780-156 Oeiras, Portugal
}

The auditory system has two parallel streams in the brain that have been implicated in auditory fear learning. The lemniscal stream has selective neurons that are tonotopically organized and is thought to be important for sound discrimination. The nonlemniscal stream has less selective neurons, which are not tonotopically organized, and is thought to be important for multimodal processing and for several forms of learning. Therefore, it has been hypothesized that the lemniscal, but not the nonlemniscal, pathway supports discriminative fear to auditory cues. To test this hypothesis we assessed the effect of electrolytic lesions to the ventral, or medial, division of the medial geniculate nucleus (MGv or MGm, which correspond, respectively, to the lemniscal and the nonlemniscal auditory pathway to amygdala) on the acquisition, expression and extinction of fear responses in discriminative auditory fear conditioning, where one tone is followed by shock (conditioned stimulus, $\mathrm{CS}^{+}$), and another is not $\left(\mathrm{CS}^{-}\right)$. Here we show that with single-trial conditioning control, $\mathrm{MGv}$ - and $\mathrm{MGm}$-lesioned male rats acquire nondiscriminative fear of both the $\mathrm{CS}^{+}$and the $\mathrm{CS}^{-}$. However, after multiple-trial conditioning, control rats discriminate between the $\mathrm{CS}^{+}$and $\mathrm{CS}^{-}$, whereas MGv- and MGm-lesioned do not. Furthermore, post-training lesions of MGm, but not MGv, lead to impaired expression of discriminative fear. Finally, MGm-lesioned rats display high levels of freezing to both the $\mathrm{CS}^{+}$ and $\mathrm{CS}^{-}$even after an extinction session to the $\mathrm{CS}^{+}$. In summary, our findings suggest that the lemniscal pathway is important for discriminative learning, whereas the nonlemniscal is important for negatively regulating fear responses.

\section{Introduction}

Learning from aversive events is conserved across species as it is crucial for survival. Furthermore, several behavioral disorders entail nonadaptive and generalized fear responses in human subjects (Grillon, 2002). Fear learning has thus been intensively studied in the past decades and interest has been growing on the mechanisms of generalization of conditioned fear (Armony et al., 1997; Shaban et al., 2006; Duvarci et al., 2009). Classical fear conditioning, where a neutral tone (conditioned stimulus, CS) is paired with an aversive footshock (unconditioned stimulus, US) after which the CS is able to elicit by itself fear responses, has been the key paradigm to study the neural mechanisms underlying this form of learning. In auditory fear conditioning (AFC), information about auditory CSs reaches the amygdala, a key structure for learning and recall of the CS-US association (LeDoux, 2000; Rumpel et al., 2005; Han et al., 2007), either directly from the nonlemniscal auditory thalamus or indirectly via auditory cortex comprising both the lemniscal and nonlemniscal pathways (Romanski and LeDoux, 1993). Furthermore, there is evidence that both the direct and indirect pathways are implicated in AFC (Ro-

\footnotetext{
Received Feb. 27, 2010; revised April 29, 2010; accepted June 2, 2010.

This work was supported by Fundação Champalimaud, Instituto Gulbenkian de Ciência and Fundação para a Ciência e a Tecnologia, Portugal (POCTI/SAU-NEU/56935/2004). R.A. was supported by Fundação para a Ciência e Tecnologia SFRH/BD/27500/2006. We thank Marta Guimarãis and Rosalina Fonseca for fruitful discussions and help with experiments, and Rui Costa, Susana Lima, and Joseph Paton for helpful comments on this manuscript.

Correspondence should be addressed to Marta A. Moita, Fundação Champalimaud Neuroscience Programme at Instituto Gulbenkian de Ciência, Rua da Quinta Grande, 6, P-2780-156 0eiras, Portugal. E-mail: moita@ igc.gulbenkian.pt.

DOI:10.1523/JNEUROSCI.1037-10.2010

Copyright $\odot 2010$ the authors $\quad 0270-6474 / 10 / 309782-06 \$ 15.00 / 0$
}

manski and Ledoux, 1992; McKernan and Shinnick-Gallagher, 1997; Rumpel et al., 2005; Rutkowski and Weinberger, 2005; Boatman and Kim, 2006). It is widely accepted that the cortical pathway ("high route") is crucial for discrimination between fearful and neutral sounds, while the direct thalamic pathway ("low route") provides a rapid but less accurate relay of auditory information to the amygdala (for review, see LeDoux, 2000). However, little evidence supports this hypothesis and a role for the thalamic pathway in differential fear responding has been previously suggested (Jarrell et al., 1986, 1987; Han et al., 2008). Hence, we aimed at investigating the role of the lemniscal and nonlemniscal pathway in auditory discrimination.

In the lemniscal pathway, sharply tuned neurons in primary auditory cortex receive their main input from the ventral division of medial geniculate nucleus (MGv), which is tonotopically organized, has narrowly tuned neurons and does not project directly to the amygdala (Bordi and Ledoux, 1994a; Kimura et al., 2003). In contrast, in the nonlemniscal pathway, the medial division of medial geniculate nucleus (MGm) has multisensory and nontuned auditory responses (for the range of frequencies in the present work, see Materials and Methods) (Bordi and LeDoux, 1994 a,b) and is the main direct input to the amygdala, although it also sends diffuse projections to auditory cortex (Kimura et al., 2003) (Fig. 1a). Therefore, we hypothesized that if the cortical pathway is engaged in auditory discrimination, it is probably via lemniscal projections from the MGv (indirect pathway), whereas MGm (direct nonlemniscal pathway) should not be required.

To test our hypothesis we assessed the effect of MGv or MGm bilateral electrolytic lesions (see Materials and Methods; Fig. 1b) on the acquisition, expression and extinction of fear responses in 
a

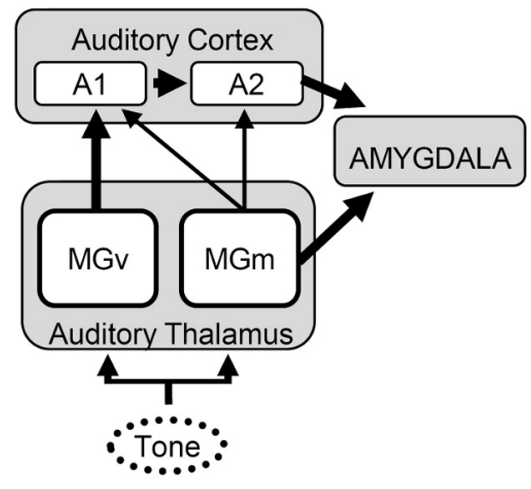

b
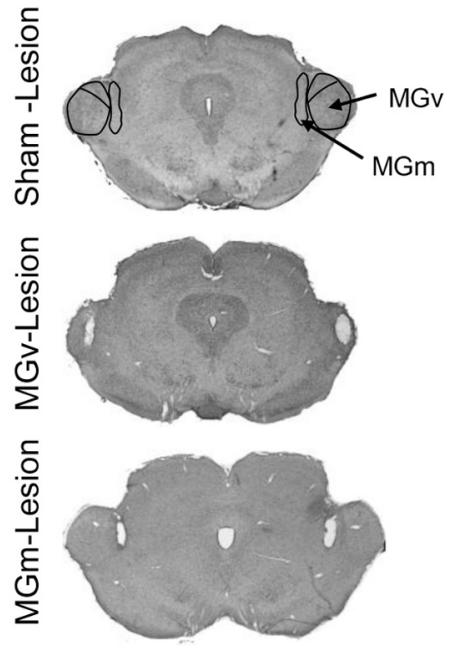

Figure 1. Neural circuit for auditory fear. $\boldsymbol{a}$, Direct and indirect auditory pathways to the amygdala. Thicker arrows represent major neuronal inputs. $\boldsymbol{b}$, Coronal sections showing example electrolytic lesions of the thalamic nuclei (for a schematic representation of the extent of the lesions see supplemental Fig. 1, available at www.jneurosci.org as supplemental material). A1, Primary auditory cortex; $\mathrm{A2}$, secondary auditory cortex.

discriminative $\mathrm{AFC}$, where one tone $\left(\mathrm{CS}^{+}\right)$was paired with shock and a second tone $\left(\mathrm{CS}^{-}\right)$was explicitly unpaired with shock. Since, in discriminative learning, animals first acquire generalized fear to both $\mathrm{CS}^{+}$and $\mathrm{CS}^{-}$and discrimination between CSs is gradually learned with extended training (Pearce, 1994), we designed two training tasks that allow studying the mechanisms underlying the acquisition of generalized and discriminative fear, and tested the effect of MGm and MGv lesions on both training schemes (Figs. 2a, 3a).

\section{Materials and Methods}

Subjects. Subjects were naive male Sprague Dawley rats $(300-450 \mathrm{~g})$ obtained from a commercial supplier (Harlan). After arrival animals were single housed in Plexiglas top filtered cages and maintained on a $12 \mathrm{~h}$ light/dark cycle (lights off at 7:00 P.M.) with ad libitum access to food and water. Rats were acclimated for at least 1 week before experimental manipulation and all animals were handled for a few days before each experiment. All behavioral and surgical procedures were performed during the light phase of the cycle.

The Instituto Gulbenkian de Ciência follows the European Guidelines. The use of vertebrate animals in research in Portugal complies with the European Directive 86/609/EEC of the European Council.

Surgery. Electrolytic lesions were performed under stereotaxic surgery. Given the small dimension of the MGm nucleus and the fact that even small lesions had an effect on discrimination, performing excitotoxic lesions was not possible since just the injector track at the injection sites was sufficient to cause an effective lesion.

Rats were anesthetized with sodium pentobarbital $(65 \mathrm{mg} / \mathrm{kg})$ and given atropine $(33 \mathrm{mg} / \mathrm{kg})$. The skin above the skull was retracted and cranial holes were made above the lesions area. Electrolytic lesions were made using stainless steel electrodes $(0.25 \mathrm{~mm}$ in diameter $)$ insulated except for $<0.5 \mathrm{~mm}$ of the tip. A constant current source (Stimulus isolator, WPI) was used for all lesions (MGv lesions: $0.75 \mathrm{~mA}, 10 \mathrm{~s} ; \mathrm{MGm}$ lesions: $0.45 \mathrm{~mA}, 6 \mathrm{~s})$. Stereotaxic coordinates relative to interaural zero according to Paxinos and Watson (1986) were used. Three penetration sites along the anteroposterior axis were used for both MGv and MGm lesions. For MGv lesions the coordinates were as follows: anterior [+3.6 $\mathrm{mm}, 3.2 \mathrm{~mm}, 2.8 \mathrm{~mm}$ ]; lateral [ $\pm 3.4 \mathrm{~mm}, 3.5 \mathrm{~mm}, 3.7 \mathrm{~mm}$; ventral $[+3.8 \mathrm{~mm},+3.7 \mathrm{~mm},+3.7 \mathrm{~mm}]$. For MGm lesions the coordinates were as follows: anterior [ $+3.7 \mathrm{~mm}, 3.2 \mathrm{~mm}, 2.7 \mathrm{~mm}$ ]; lateral [ $\pm 2.5 \mathrm{~mm}, 2.6$ $\mathrm{mm}, 2.9 \mathrm{~mm}$ ]; ventral $[+3.7 \mathrm{~mm},+3.7 \mathrm{~mm},+3.6 \mathrm{~mm}]$. In sham surgeries the electrode was placed $1.0 \mathrm{~mm}$ above the ventral coordinate without passing current. Once all penetrations were done, the holes in

the skull were covered with sterile Vaseline and the skin was sutured. A single subcutaneous injection of the analgesic buprenorphine $(0.02$ $\mathrm{mg} / \mathrm{kg}$ ) was given postsurgically.

For the pre-training lesion experiments, animals were allowed to recover for 1 week after surgery before training begun. For the posttraining lesion experiments, surgeries were performed $24 \mathrm{~h}$ after the last training session. The animals were then allowed to recover for 1 week, after which the discrimination test session took place.

Behavior. Two distinct environments (A and B) were used in this study. These two environments were located in the same procedure room and were used in a counterbalanced manner (i.e., the animals conditioned in A were tested in $B$ and vice versa). Both consisted of one conditioning chamber (model H1011R-TC, Coulbourn Instruments) inside a high sound-attenuating cubicle lined with dark gray decoupling foam (model H10-24A, Coulbourn Instruments). During training, both chambers had a shock floor of metal bars (model H10-11R-TC-SF, Coulbourn Instruments), but during test sessions, the floor in both chambers was covered by a painted acrylic floor. To minimize generalization between the two environments, several features of the environments differed. In box A, the ceiling and all four side walls were made of clear Plexiglas and the sound-attenuating cubicle was lined with yellow paper. The house light was in the middle-top of the left wall and the speaker was placed outside the chamber, behind the right wall. In Box B, the two side walls were made of polished sheet metal. The house light was red and placed in the top-back corner of the right wall and the speaker was behind the left wall. Furthermore, the boxes were cleaned with two different detergents.

The tones were produced by a sound generator (RM1, Tucker-Davis Technologies) and delivered through a horn tweeter (model TL16H8OHM, VISATON). The sound was calibrated using a Brüel and Kjaer microphone (type 4189) and sound analyzer (hand held analyzer type 2250). A precision programmable shocker (model H13-16, Coulbourn Instruments) delivered footshocks. A video camera mounted on the ceiling of each attenuating cubicle recorded the rats' behavior. A surveillance video acquisition system was used to store all video in hard disk for posterior off line scoring of freezing behavior by blind observers with timers.

Experiment 1-role of $M G v$ and $M G m$ in acquisition of auditory fear conditioning. Animals were subjected to one of two training protocols: single or multiple trial conditioning. Single trial conditioning consisted of one single presentation of the $\mathrm{CS}^{+}$coterminating with a footshock $(0.5 \mathrm{~mA}, 0.5 \mathrm{~s})$, followed by a single presentation of the $\mathrm{CS}^{-}$, with an 180 s intertrial interval (ITI). Multiple trial conditioning consisted of two sessions, each comprising 4 random presentations of the $\mathrm{CS}^{+}$, which coterminated with a footshock $(0.5 \mathrm{~mA}, 0.5 \mathrm{~s})$, and 4 random presentations of the $\mathrm{CS}^{-}$, with an average $180 \mathrm{~s}$ ITI. In both protocols the $\mathrm{CS}^{+}$ was a $10 \mathrm{kHz}$ pure tone $(60 \mathrm{~dB}, 20 \mathrm{~s})$ and $\mathrm{CS}^{-}$was a $2 \mathrm{kHz}$ pure tone $(60$ $\mathrm{dB}, 20 \mathrm{~s}$ ). These tone frequencies were chosen as they lie in the region of the auditory spectrum to which both neurons in amygdala and MGm show flat receptive fields, i.e., no discriminative firing in naive animals can be observed. Neurons in these structures show selective firing for higher frequency ranges (Bordi and LeDoux, 1994a,b), which correspond to social ultrasonic vocalizations (USVs). As we are interested in general mechanisms of discrimination we chose to avoid frequencies that are close to the ones used in social communication. Furthermore, it has been shown that rats trained in a differential $\mathrm{CS}^{+} / \mathrm{CS}^{-} \mathrm{AFC}$ protocol tend to generalize their fear responses toward $22 \mathrm{kHz}$ tones, which corresponds to the fundamental frequency of alarm calls (Bang et al., 2008). Thus, the frequency of the $\mathrm{CS}^{+}$was chosen to be the $10 \mathrm{kHz}$ frequency (which is closer to the $22 \mathrm{kHz}$ principal frequency), to avoid biasing our 


\section{a Single Trial Conditioning}

$\mathrm{CS}^{+}(10 \mathrm{KHz}, 60 \mathrm{~dB}, 20 \mathrm{sec}) \quad \mathrm{CS}^{-}(2 \mathrm{KHz}, 60 \mathrm{~dB}, 20 \mathrm{sec})$
US $(0.5 \mathrm{~mA}, 0.5 \mathrm{sec})$

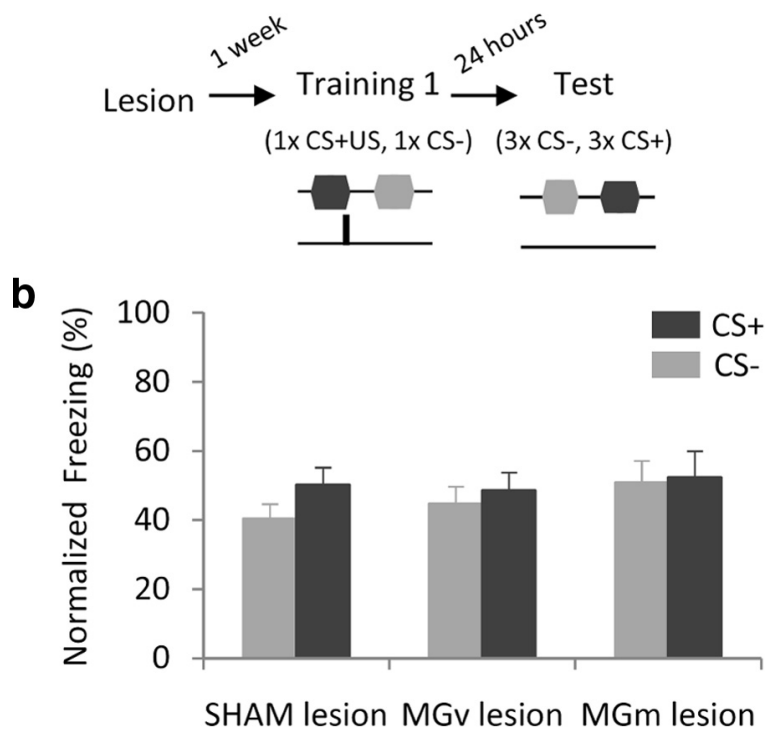

Figure 2. Each pathway is sufficient for the acquisition of generalized fear. $\boldsymbol{a}$, Schematic showing experimental protocol. $\boldsymbol{b}$, Freezing responses to $\mathrm{CS}^{+}$and $\mathrm{CS}^{-}$during discrimination test. Freezing responses are presented as percentage freezing normalized to baseline freezing levels (for raw data see supplemental Fig. 2, available at www.jneurosci.org as supplemental material). Data are presented as mean \pm SEM for $n=24$ animals (control, $n=11$; MGv-lesion, $n=8 ;$ MGm-lesion, $n=5$ ).

results toward generalization, which may arise from responses to USVs and innate fear.

Rats were tested for their fear of the $\mathrm{CS}^{+}$and $\mathrm{CS}^{-} 24 \mathrm{~h}$ after the last training session. The same testing protocol was used for rats subjected to the single and multiple trial conditioning protocols. Testing took place in a box differing from the training box in a number of cues (including texture, color, and odor of the chamber, see above). Three presentations of the $\mathrm{CS}^{-}$were followed by 3 presentations of the $\mathrm{CS}^{+}$, with a 5 min ITI.

Experiment $2 a$-role of $M G v$ or $M G m$ in the recall of discriminative auditory fear conditioning. Intact animals were trained using the multiple-trial conditioning protocol of experiment 1 . Lesions were performed $1 \mathrm{~d}$ after the last training session. Rats were allowed to recover from surgery and tested for their fear of the $\mathrm{CS}^{+}$and $\mathrm{CS}^{-} 1$ week after surgery. As in experiment 1 , testing took place in a different box and the same testing protocol was used.

Experiment $2 b$-role of $M G v$ or $M G m$ in the extinction of previously learned discriminative fear responses. For this experiment a subset of animals from the three groups (sham, MGv, and MGm lesion) of experiment 2 was used. One day after the discrimination test, these animals underwent an extinction session (10 presentations of the $\left.\mathrm{CS}^{+}\right)$in the testing box. One day later, freezing to the $\mathrm{CS}^{-}$and the $\mathrm{CS}^{+}$was retested, using the same testing protocol as before. Thus, even though animals were conditioned with an intact brain, extinction training was performed in lesioned animals.

Histology. At the end of each experiment all the animals were deeply anesthetized with an overdose of sodium pentobarbital and transcardially perfused with $1 \%$ PBS salt solution followed by $10 \%$ formalin solution (Sigma). After this, the brains were removed and stored in refrigerator in a $30 \%$ sucrose/formalin postfix solution until they sank (2-3 d). Then, $40-\mu \mathrm{m}$-thick coronal sections covering the whole extent of the MGm or MGv areas were cut on a cryostat. Every third section was collected on coated slides and stained with cresyl violet. Sections were then examined in a light microscope to confirm location and extent of the lesioned area.
For all experiments, from a total of $31 \mathrm{MGv}$-lesions performed, 16 were excluded, and from a total of $57 \mathrm{MGm}$-lesions performed, 37 were also excluded due to misplacement, small extent, or lesions extending to the neighboring nuclei (supplemental Fig. 1, available at www.jneurosci. org as supplemental material, shows the extent of the smallest and biggest lesions of MGm and MGv included in this study).

Statistical analysis. Freezing scores correspond to the duration of time spent freezing at specific time periods: before any CS was presented $(20 \mathrm{~s}$ baseline) and during each $\mathrm{CS}^{+}$and $\mathrm{CS}^{-}$. In all experiments freezing scores during the $\mathrm{CS}^{+}$and the $\mathrm{CS}^{-}$were normalized to the baseline (for each animal the difference between freezing during each CS and baseline was calculated), so that differences between freezing scores during the $\mathrm{CS}^{+}$and $\mathrm{CS}^{-}$do not reflect individual differences in baseline fear. Animals with a high baseline freezing score were excluded [above 50\%, corresponding to abnormal values which were defined by: freezing score $>$ third quartile $+1.5 *$ (third quartile - first quartile) $]$. Importantly, no differences in baseline fear were found between groups in any of the experiments (see supplemental Figs. 2-4, available at www. jneurosci.org as supplemental material) (Kruskal-Wallis tests: experiment 1 , single trial conditioning, $K_{(2)}=3.06, p=0.22$; experiment 1 , multiple trial, $K_{(2)}=3.73, p=0.16$; experiment $2, K_{(2)}=1.12, p=0.57$; experiment $\left.3, K_{(2)}=1.89, p=0.39\right)$.

Because not all variables followed a normal distribution, homoscedasticity is not met and due to the small sample size, we used nonparametric statistics. Discrimination was assessed by testing whether freezing evoked by the $\mathrm{CS}^{+}$was higher than that triggered by the $\mathrm{CS}^{-}$. To this end, one-tailed Wilcoxon ranked signed tests, performed within groups and Bonferroni corrected for multiple comparisons (critical value, $\alpha=0.0167$ ) were used. All analyses were performed using the statistical software XLSTAT (Microsoft).

\section{Results}

\section{MGv and MGm are required for acquisition of discriminative} auditory fear conditioning

Consistent with previous findings, sham animals acquired fear of the $\mathrm{CS}^{+}$and generalized to the $\mathrm{CS}^{-}$after single trial conditioning (Fig. 2; no significant difference between freezing to the $\mathrm{CS}^{+}$and the $\mathrm{CS}^{-}, V=24.0$ and $p=0.23$ ). Similarly, MGv- and MGmlesioned rats showed intact acquisition of generalized fear responses after single trial conditioning (Fig. 2; no significant difference between $\mathrm{CS}^{+}$and $\mathrm{CS}^{-}$-elicited freezing was observed: $V=16.0$ and $p=0.42, V=7.0$ and $p=0.50$, respectively). Thus, both pathways are sufficient for single trial-conditioning, which entails conditioned fear generalized to the $\mathrm{CS}^{-}$.

Multiple trial conditioning lead to the acquisition of discriminative freezing in control animals (Fig. 3; freezing to the $\mathrm{CS}^{+}$was significantly higher than to the $\mathrm{CS}^{-}, V=0.0$ and $p=0.0005$ for the sham-lesioned group). In contrast, $\mathrm{MGv}$ lesioned animals failed to discriminate between $\mathrm{CS}^{+}$and $\mathrm{CS}^{-}$ after multiple trial conditioning (Fig. 3; again freezing during the $\mathrm{CS}^{+}$was not significantly different from freezing during the $\mathrm{CS}^{-}, V=5.0$ and $p=0.08$, note that the critical value $\alpha$ is $0.0167)$. Furthermore, we found that MGm-lesioned rats also failed to discriminate between $\mathrm{CS}^{+}$and $\mathrm{CS}^{-}$after multiple trial conditioning (Fig. 3; no significant difference between freezing to the $\mathrm{CS}^{+}$and $\mathrm{CS}^{-}$was found, $V=6.5$ and $p=0.45)$. Importantly, this result cannot be explained by a possible disruption of MGv projections that may cross MGm, as this would correspond to a combined lesion of MGv (fibers) and MGm (cell bodies), which should lead to impaired auditory-evoked freezing (LeDoux et al., 1983). Therefore, both the lemniscal and the nonlemniscal pathways are required for intact discriminative fear acquisition. 
MGm, but not MGv, is required for the recall of discriminative fear

As expected, in this experiment sham animals were able to express discriminative freezing between the $\mathrm{CS}^{-}$and the $\mathrm{CS}^{+}$, showing significantly higher levels of freezing to the $\mathrm{CS}^{+}$than the $\mathrm{CS}^{-}$(Fig. 4b, $V=0.0$ and $p=0.004)$. Similarly, MGv-lesioned rats showed clear discriminative freezing between the $\mathrm{CS}^{+}$and the $\mathrm{CS}^{-}$(Fig. $4 b, V=$ 2.0 and $p=0.0005$ ). In contrast, even though all groups were trained with an intact brain and, thus, acquired normally discriminative fear, MGm-lesioned animals showed impaired expression of discriminative fear, showing similar levels of freezing to the $\mathrm{CS}^{+}$and $\mathrm{CS}^{-}$(Fig. $4 b, V=12.0$ and $p=0.12$ ). Furthermore, the magnitude of freezing observed in these animals was comparable to that of $\mathrm{CS}^{+}$-evoked freezing in either sham or MGv-lesioned groups. Hence, generalization between $\mathrm{CS}^{+}$and $\mathrm{CS}^{-}$in $\mathrm{MGm}$-lesioned animals seems to arise from an inability to suppress freezing to the $\mathrm{CS}^{-}$.

\section{MGm, but not MGv, is required for fear extinction}

During the discrimination test, $1 \mathrm{~d}$ after the extinction session consisting of repeated presentations of the $\mathrm{CS}^{+}$alone, sham animals showed decreased levels of freezing to the $\mathrm{CS}^{+}\left(\mathrm{CS}^{+}\right.$before extinction: $70.0 \pm 3.9$; $\mathrm{CS}^{+}$after extinction: $23.3 \pm 5.6$ ), which was comparable to freezing to the $\mathrm{CS}^{-}$(Fig. $4 c$, no difference between $\mathrm{CS}^{+}$- and $\mathrm{CS}^{-}$-evoked freezing was observed in the discrimination test after extinction, $V=4.0$ and $p=0.44)$. MGvlesioned rats also extinguished freezing to the $\mathrm{CS}^{+}\left(\mathrm{CS}^{+}\right.$before extinction: $65.3 \pm 3.6$; $\mathrm{CS}^{+}$after extinction: $40.5 \pm 6.6$ ). However, in contrast with control animals, MGv-lesioned rats seemed to display discriminative freezing during the post-extinction discrimination test (Fig. $4 c$, during the post-extinction discrimination test, a trend for freezing to the $\mathrm{CS}^{+}$being higher than freezing to the $\mathrm{CS}^{-}$was observed, $V=2.0$ and $p=0.023$ ). Finally, MGm-lesioned rats showed impaired recall of extinction memory of the $\mathrm{CS}^{+}\left(\mathrm{CS}^{+}\right.$ before extinction: 70.2 \pm 6.1 ; $\mathrm{CS}^{+}$after extinction: $65.0 \pm 7.1$ ).

Consistent with the finding that these animals failed to show discriminative fear during the pre-extinction session, in the postextinction discrimination test they also expressed high levels of freezing to the $\mathrm{CS}^{-}$, comparable to freezing evoked by the $\mathrm{CS}^{+}$ (Fig. 4c; no difference between $\mathrm{CS}^{+}$- and $\mathrm{CS}^{-}$-evoked freezing was observed in the discrimination test after extinction, $V=2.5$ and $p=0.112$ ).

Finally, confirming the effect of MGm lesions on fear extinction, a Kruskal-Wallis test revealed a significant effect of group on freezing to the $\mathrm{CS}^{+}$after extinction $\left(K_{(2)}=7.5, p=0.024\right)$. Post hoc comparisons showed that MGm-, but not MGv-lesioned, animals froze significantly more than control animals to the $\mathrm{CS}^{+}$after extinction $(\alpha=0.0167)$.

\section{Discussion}

The present study confirms the hypothesis that the indirect lemniscal auditory pathway to the amygdala is necessary for normal discrimination (for review, see LeDoux, 2000), and shows in addition that discrimination also relies on the direct nonlemniscal pathway. Furthermore, we show that even though both pathways are required for intact discriminative learning, their contribution is likely to rely on different mechanisms.

The finding that both MGv-lesioned and MGm-lesioned rats show intact acquisition of generalized fear responses after single trial conditioning is consistent with a previous report showing that neither cortical nor MGm lesions affect acquisition of fear of a tone paired with shock (Romanski and LeDoux, 1992). This redundancy in neuronal pathways involved in the acquisition of

\section{a Multiple Trial Conditioning}

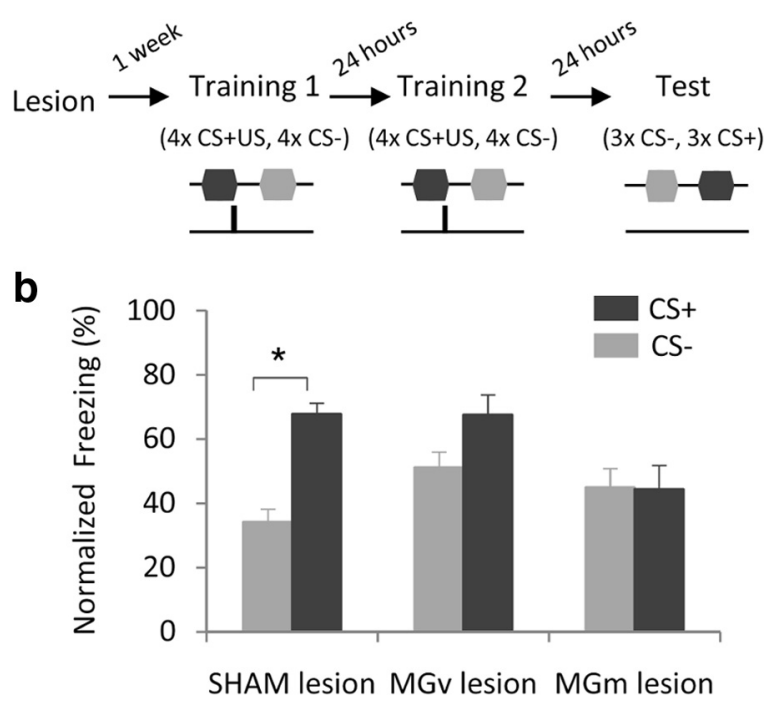

Figure 3. Both pathways are necessary for the acquisition of discriminative fear. $\boldsymbol{a}$, Schematic showing experimental protocol. $\boldsymbol{b}$, Freezing responses to $\mathrm{CS}^{+}$and $\mathrm{CS}^{-}$during discrimination test. Freezing responses are presented as percentage freezing normalized to baseline freezing levels (for raw data see supplemental Fig. 3, available at www.jneurosci.org as supplemental material). Data are presented as mean \pm SEM for $n=25$ animals (control, $n=14$; MGv-lesion, $n=7$; MGm-lesion, $n=5$ ). ${ }^{*} p<0.0167$.

fear may guarantee self-preservation, even though in single trial learning the learned fear generalizes to other auditory stimuli. On the other hand, discriminative fear learning, achieved with multiple trials, requires activity of the two coexisting pathways. The finding that MGv-lesioned rats fail to discriminate after multiple trial conditioning supports the hypothesis that the indirect lemniscal pathway is crucial for auditory discrimination (LeDoux, 2000). MGv may be important for discriminative learning by facilitating cortical retuning (Ma and Suga, 2009), which might enhance the contrast between $\mathrm{CS}^{+}$- and $\mathrm{CS}^{-}$-evoked activity in $\mathrm{AC}$, leading in turn to an increase in the $\mathrm{CS}^{+}$-elicited freezing.

It has previously been shown that rats with auditory cortex (AC) lesions, when trained to a single pure tone that is paired with footshock, and tested for their fear of tones of different frequencies, show a generalization gradient similar to that of control unlesioned rats (Armony et al., 1997). This finding was taken as indicative that the auditory cortex was not important for discriminative fear. One possible explanation for the discrepancy between this previous study and our findings is that we trained rats in a discriminative protocol, which may render this task dependent on the indirect cortical pathway, via the MGv nucleus. Moreover, in concordance with the present work, earlier studies have shown that either AC or MGm lesions seem to impair acquisition and expression of differential bradycardia (Jarrell et al., 1986, 1987; Teich et al., 1988). However, analogies made between conditioned freezing and heart rate changes must be taken cautiously, since these seem to rely on different mechanisms as illustrated by the fact that the acquisition of conditioned heart rate changes, but not conditioned freezing, to an auditory cue depends on an intact MGm (McCabe et al., 1993).

The finding that MGm lesions also impair discriminative fear learning is at contrast with the high route/low route hypothesis, 
which states that the indirect lemniscal, but not the direct nonlemniscal, pathway is important for auditory discrimination. Our present findings also show that when discrimination is normally learned, expression of the learned discriminative fear responses is impaired by $\mathrm{MGm}$, but not MGv lesions. Thus, although for acquisition of discriminative fear both the lemniscal and nonlemniscal auditory pathways are necessary, its recall seems to rely solely on the latter. In addition, posttraining lesions of the direct nonlemniscal thalamic pathway result in expression of high freezing levels to both the reinforced and the nonreinforced auditory stimuli, suggesting that this pathway might be important for discriminative fear by suppressing freezing to the neutral cues.

Consistent with a suppressive role of the direct thalamic input to the amygdala in discriminative fear, we found that MGm lesions impaired the expression of extinguished fear. This result is consistent with recent findings that the medial geniculate nucleus (including both MGm and MGv) is required for the recall of extinction memory (Orsini and Maren, 2009). Extinction is thought to be a process of relearning that depends on the inhibition of previously learned responses (for review, see Ehrlich et al., 2009). Therefore, the effect of MGm-lesions on this learning process might result from a disruption of the inhibitory drive onto the amygdala, which is consistent with a significant feedforward inhibition from the thalamus to the amygdala (Shaban et al., 2006; Shin et al., 2006; Pan et al., 2009). This may also be the mechanism by which the MGm is involved in the recall of discriminative fear, which as extinction seems to depend on the ability to inhibit freezing, in this case to the $\mathrm{CS}^{-}$. The MGm may play a role in discriminative fear either by suppressing freezing specifically to the $\mathrm{CS}^{-}$or by providing tonic inhibition to the amygdala, working as a gate which only strong inputs like the $\mathrm{CS}^{+}$could surpass. Indeed the amygdala is under tight inhibitory control (Shin et al., 2006; Ehrlich et al., 2009; Pan et al., 2009; Amano et al., 2010) and disruption of presynaptic inhibition has been shown to lead to generalization of fear responses (Shaban et al., 2006).

In summary, in the present study we show, on the one hand, that the lemniscal input pathway to the amygdala is necessary for normal acquisition, but not recall, of discriminative fear, possibly by enhancing the representation of relevant relative to the irrelevant cues (Gao and Suga, 2000; Rutkowski and Weinberger, 2005; Weinberger, 2007). On the other hand, our results suggest that the nonlemniscal pathway is important to suppress fear of neutral or safe auditory stimuli, thereby affecting the acquisition and recall of discriminative fear (as it involves suppression of fear of the $\mathrm{CS}^{-}$) as well as the extinction of fear of an auditory cue that was previously paired with shock. Thus, this work sheds new light into the mechanisms of fear learning and may impact on the understanding of pathological states entailing mal-adaptive fear responses.

\section{b Pre-extinction Test}

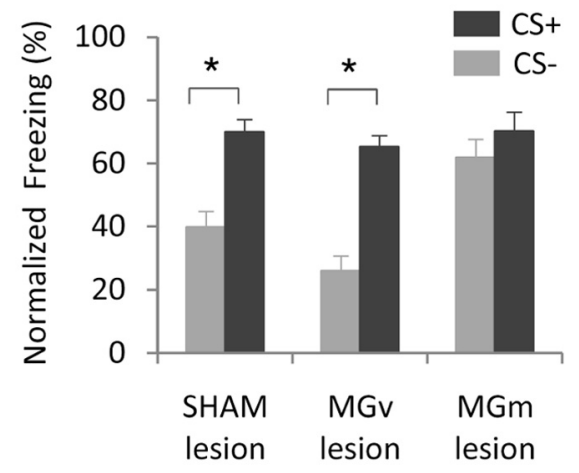

C Post-extinction Test
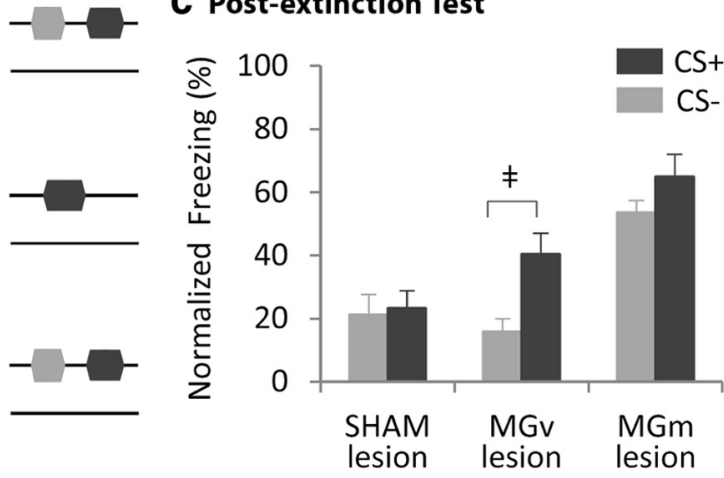

Figure 4. MGm, but not the MGv, is necessary for the expression of discriminative fear and for extinction of fear to the $\mathrm{CS}^{+} . \boldsymbol{a}_{\text {, }}$ Schematic showing experimental protocol. $\boldsymbol{b}$, Freezing responses to $\mathrm{CS}^{+}$and $\mathrm{CS}^{-}$during discrimination test, after lesion. $\boldsymbol{c}$ ercentage freezing normalized to baseline freezing levels (for raw data see supplemental Fig. 4 , available at www.jneurosci.org as supplemental material). Data are presented as mean \pm SEM for $n=29$ animals (pre-extinction: control, $n=8$; MGv-lesion, $n=12$; MGm-lesion, $n=9$; post-extinction: control, $n=4$; MGv-lesion, $n=7$; MGm-lesion, $n=5$ ). ${ }^{*} p<0.0167 ;{ }^{\ddagger} p<0.05$.

\section{References}

Amano T, Unal CT, Paré D (2010) Synaptic correlates of fear extinction in the amygdala. Nat Neurosci 13:489-494.

Armony JL, Servan-Schreiber D, Romanski LM, Cohen JD, LeDoux JE (1997) Stimulus generalization of fear responses: effects of auditory cortex lesions in a computational model and in rats. Cereb Cortex 7:157-165.

Bang SJ, Allen TA, Jones LK, Boguszewski P, Brown TH (2008) Asymmetrical stimulus generalization following differential fear conditioning. Neurobiol Learn Mem 90:200-216.

Boatman JA, Kim JJ (2006) A thalamo-cortico-amygdala pathway mediates auditory fear conditioning in the intact brain. Eur J Neurosci 24:894-900.

Bordi F, LeDoux JE (1994a) Response properties of single units in areas of rat auditory thalamus that project to the amygdala. I. Acoustic discharge patterns and frequency receptive fields. Exp Brain Res 98:261-274.

Bordi F, LeDoux JE (1994b) Response properties of single units in areas of rat auditory thalamus that project to the amygdala. II. Cells receiving convergent auditory and somatosensory inputs and cells antidromically activated by amygdala stimulation. Exp Brain Res 98:275-286.

Duvarci S, Bauer EP, Paré D (2009) The bed nucleus of the stria terminalis mediates interindividual variations in anxiety and fear. J Neurosci 29: 10357-10361.

Ehrlich I, Humeau Y, Grenier F, Ciocchi S, Herry C, Lüthi A (2009) Amygdala inhibitory circuits and the control of fear memory. Neuron 62:757-771.

Gao E, Suga N (2000) Experience-dependent plasticity in the auditory cortex and the inferior colliculus of bats: role of the corticofugal system. Proc Natl Acad Sci U S A 97:8081-8086.

Grillon C (2002) Startle reactivity and anxiety disorders: aversive conditioning, context, and neurobiology. Biol Psychiatry 52:958-975.

Han JH, Kushner SA, Yiu AP, Cole CJ, Matynia A, Brown RA, Neve RL, Guzowski JF, Silva AJ, Josselyn SA (2007) Neuronal competition and selection during memory formation. Science 316:457-460. 
Han JH, Yiu AP, Cole CJ, Hsiang HL, Neve RL, Josselyn SA (2008) Increasing CREB in the auditory thalamus enhances memory and generalization of auditory conditioned fear. Learn Mem 15:443-453.

Jarrell TW, Gentile CG, McCabe PM, Schneiderman N (1986) The role of the medial geniculate region in differential Pavlovian conditioning of bradycardia in rabbits. Brain Res 374:126-136.

Jarrell TW, Gentile CG, Romanski LM, McCabe PM, Schneiderman N (1987) Involvement of cortical and thalamic auditory regions in retention of differential bradycardiac conditioning to acoustic conditioned stimuli in rabbits. Brain Res 412:285-294.

Kimura A, Donishi T, Sakoda T, Hazama M, Tamai Y (2003) Auditory thalamic nuclei projections to the temporal cortex in the rat. Neuroscience 117:1003-1016.

LeDoux JE (2000) Emotion circuits in the brain. Annu Rev Neurosci 23:155-184.

LeDoux JE, Sakaguchi A, Reis DJ (1983) Subcortical efferent projections of the medial geniculate nucleus mediat e emotional responses conditioned to acoustic stimuli. J Neurosci 4:683-698.

Ma X, Suga N (2009) Specific and nonspecific plasticity of the primary auditory cortex elicited by thalamic auditory neurons. J Neurosci 29:4888-4896.

McCabe PM, McEchron MD, Green EJ, Schneiderman N (1993) Electrolytic and ibotenic acid lesions of the medial subnucleus of the medial geniculate prevent the acquisition of classically conditioned heart rate to a single acoustic stimulus in rabbits. Brain Res 619:291-298.

McKernan MG, Shinnick-Gallagher P (1997) Fear conditioning induces a lasting potentiation of synaptic currents in vitro. Nature 390:607-611.

Orsini CA, Maren S (2009) Glutamate receptors in the medial geniculate nucleus are necessary for expression and extinction of conditioned fear in rats. Neurobiol Learn Mem 92:581-589.

Pan BX, Dong Y, Ito W, Yanagawa Y, Shigemoto R, Morozov A (2009) Se- lective gating of glutamatergic inputs to excitatory neurons of amygdala by presynaptic GABAb receptor. Neuron 61:917-929.

Paxinos G, Watson C (1986) The rat brain in stereotaxic coordinates. Sydney: Academic.

Pearce JM (1994) Discrimination and categorization. In: Animal learning and cognition, Ed 2 (Mackintosh NJ, ed), pp 109-134. San Diego: Academic.

Romanski LM, LeDoux JE (1992) Equipotentiality of thalamo-amygdala and thalamo-cortico-amygdala circuits in auditory fear conditioning. J Neurosci 12:4501-4509.

Romanski LM, LeDoux JE (1993) Information cascade from primary auditory cortex to the amygdala: corticocortical and corticoamygdaloid projections of temporal cortex in the rat. Cereb Cortex 3:515-532.

Rumpel S, LeDoux J, Zador A, Malinow R (2005) Postsynaptic receptor trafficking underlying a form of associative learning. Science 308:83-88.

Rutkowski RG, Weinberger NM (2005) Encoding of learned importance of sound by magnitude of representational area in primary auditory cortex. Proc Natl Acad Sci U S A 102:13664-13669.

Shaban H, Humeau Y, Herry C, Cassasus G, Shigemoto R, Ciocchi S, Barbieri S, van der Putten H, Kaupmann K, Bettler B, Lüthi A (2006) Generalization of amygdala LTP and conditioned fear in the absence of presynaptic inhibition. Nat Neurosci 9:1028-1035.

Shin RM, Tsvetkov E, Bolshakov VY (2006) Spatiotemporal asymmetry of associative synaptic plasticity in fear conditioning pathways. Neuron 52:883-896.

Teich AH, McCabe PM, Gentile CG, Jarrell TW, Winters RW, Liskowsky DR, Schneiderman N (1988) Role of auditory cortex in the acquisition of differential heart rate conditioning. Physiol Behav 44:405-412.

Weinberger NM (2007) Associative representational plasticity in the auditory cortex: a synthesis of two disciplines. Learn Mem 14:1-16. 